\title{
Study of interference effects in the ${ }^{18} \mathbf{F}(p, \alpha){ }^{15} \mathbf{O}$ reaction
}

\author{
K. Y. Chae*, M. W. Guidry, C.D. Nesaraja, Z. Ma \\ Department of Physics and Astronomy, University of Tennessee, Knoxville, Tennessee 37996, \\ USA \\ Physics Division, Oak Ridge National Laboratory, Oak Ridge, Tennessee 37831, USA
}

D. W. Bardayan, J. C. Blackmon, M. S. Smith

Physics Division, Oak Ridge National Laboratory, Oak Ridge, Tennessee 37831, USA

D. Gregory, R. L. Kozub, S. Paulauskas, J. F. Shriner, Jr., N. Smith

Department of Physics, Tennessee Technological University, Cookeville, TN 38505, USA

\section{S. Johnson}

Oak Ridge Associated Universities, Bldg 6008, P. O. Box 2008, Oak Ridge, Tennessee 37831, USA

\section{R. J. Livesay, M. Porter-Peden}

Department of Physics, Colorado School of Mines, Golden, Colorado 80401, USA

\section{S. D. Pain, J. S. Thomas}

Department of Physics and Astronomy, Rutgers University, Piscataway, New Jersey 08854, USA

The ${ }^{18} \mathrm{~F}(p, \alpha){ }^{15} \mathrm{O}$ reaction plays a crucial role in understanding $\gamma$-ray emission from novae. Because of the importance of understanding the ${ }^{18} \mathrm{~F}+p$ reactions, a number of studies of the $\mathrm{A}=19$ isobars have been made using stable and exotic beams. The interference effects among $J^{\pi}=\frac{3}{2}^{+}$ resonances in the ${ }^{18} F+p$ system, however, have never been measured, but they can change the $\mathrm{S}$-factor by a factor of 20 at nova energies. $R$-matrix calculations indicate that the cross sections above the $E_{c . m .}=665 \mathrm{keV}$ resonance are sensitive to the interference between the $E_{c . m .}=$ 8,38 , and $665 \mathrm{keV}$ resonances. In order to study the interference effects, an excitation function for the ${ }^{1} \mathrm{H}\left({ }^{18} \mathrm{~F}, \alpha\right){ }^{15} \mathrm{O}$ reaction has been measured in the energy range of $E_{c . m} .=663-877 \mathrm{keV}$ using radioactive ${ }^{18} \mathrm{~F}$ beams at the Holifield Radioactive Ion Beam Facility. By measuring the ${ }^{18} \mathrm{~F}(p, \alpha){ }^{15} \mathrm{O}$ cross section off resonance and comparing the cross section with theoretical calculations, we provide the first experimental constraints on the interference of $\frac{3}{2}^{+}$resonances.

International Symposium on Nuclear Astrophysics - Nuclei in the Cosmos - IX

June 25-30 2006

CERN, Geneva, Switzerland 
The decay of radioactive ${ }^{18} \mathrm{~F}$ nuclei in the expanding envelope of novae is the most important positron annihilation source of $\gamma$-rays during the first several hours after the expansion $[1,2]$. The ${ }^{18} \mathrm{~F}(p, \alpha){ }^{15} \mathrm{O}$ reaction plays a crucial role in understanding the destruction of ${ }^{18} \mathrm{~F}$ that is synthesized in novae via proton capture on ${ }^{17} \mathrm{O}$ or through the sequence ${ }^{17} \mathrm{~F}(p, \gamma){ }^{18} \mathrm{Ne}\left(\mathrm{e}^{+} v_{e}\right){ }^{18} \mathrm{~F}$ [3]. Although a number of studies on the ${ }^{18} \mathrm{~F}+p$ system have substantially improved our understanding of the ${ }^{18} \mathrm{~F}(p, \alpha){ }^{15} \mathrm{O}$ reaction [3-11], the interference among $J^{\pi}=3 / 2^{+}$resonances could not be taken into account in the reaction rate calculations due to the lack of experimental knowledge about the relative signs of the effect. These interference effects can, however, change the astrophysical $\mathrm{S}$-factor by up to a factor of 20 at nova energies.

The ${ }^{1} \mathrm{H}\left({ }^{18} \mathrm{~F}, \alpha\right){ }^{15} \mathrm{O}$ excitation function was measured over the energy range $E_{c . m} . \simeq 663$ $877 \mathrm{keV}$ at the Oak Ridge National Laboratory (ORNL) Holifield Radioactive Ion Beam Facility (HRIBF) [12]. The production of ${ }^{18} \mathrm{~F}$ radioactive ion beams at HRIBF is based on the Isotope Separation On-Line (ISOL) technique [13]. A beam of ${ }^{4} \mathrm{He}(\sim 1 \mu \mathrm{A}, 85 \mathrm{MeV})$ from the Oak Ridge Isochronous Cyclotron (ORIC) bombarded a thick $\mathrm{HfO}_{2}$ target to produce ${ }^{18} \mathrm{~F}$ atoms via ${ }^{16} \mathrm{O}(\alpha, p n){ }^{18} \mathrm{~F}$ reaction [14]. The ${ }^{18} \mathrm{~F}$ atoms were then mass analyzed and post accelerated by the tandem electrostatic accelerator to the appropriate energies for this experiment. A schematic diagram of the experimental setup is shown in Figure 1. A beam of ${ }^{18} \mathrm{~F} /{ }^{18} \mathrm{O}$ impinged on a $70 \mu \mathrm{g} / \mathrm{cm}^{2}$ polypropylene $\mathrm{CH}_{2}$ target $\left(5.5 \times 10^{18}{ }^{1} \mathrm{H}\right.$ atoms $\left./ \mathrm{cm}^{2}\right)$. The average ${ }^{18} \mathrm{~F}$ current was $\sim 10^{5}$ ions per second, and total of $4 \times 10^{10}{ }^{18} \mathrm{~F}$ ions were delivered to the target during the experiments. Energy steps of $\Delta E_{c . m}$. $\simeq 50 \mathrm{keV}\left(\Delta E_{\text {lab }}=1 \mathrm{MeV}\right)$ were taken because the ${ }^{18} \mathrm{~F}$ ions lose about $970 \mathrm{keV}$ in the target at this energy range. The recoil particles from the ${ }^{1} \mathrm{H}\left({ }^{18} \mathrm{~F}, \alpha\right){ }^{15} \mathrm{O}$ reaction $(\alpha$ particles and ${ }^{15} \mathrm{O}$ ions) were detected in coincidence by two large area silicon detector arrays. The ${ }^{18} \mathrm{~F}$ and ${ }^{18} \mathrm{O}$ ions which were scattered from the carbon component of the $\mathrm{CH}_{2}$ target were also continuously detected by a gas-filled ionization counter enabling a constant monitor of the beam composition. The cross sections measured in this experiment are plotted in Figure 2.

To study the interference effects on the cross section, the $R$-matrix code MULTI [15] was used. The free parameters were the signs of three $J^{\pi}=3 / 2^{+}$resonance terms for the levels at $E_{c . m .}=8,38$, and $665 \mathrm{keV}$, where we use the sign convention adopted in Eq. (XII. 5. 15) of Lane and Thomas

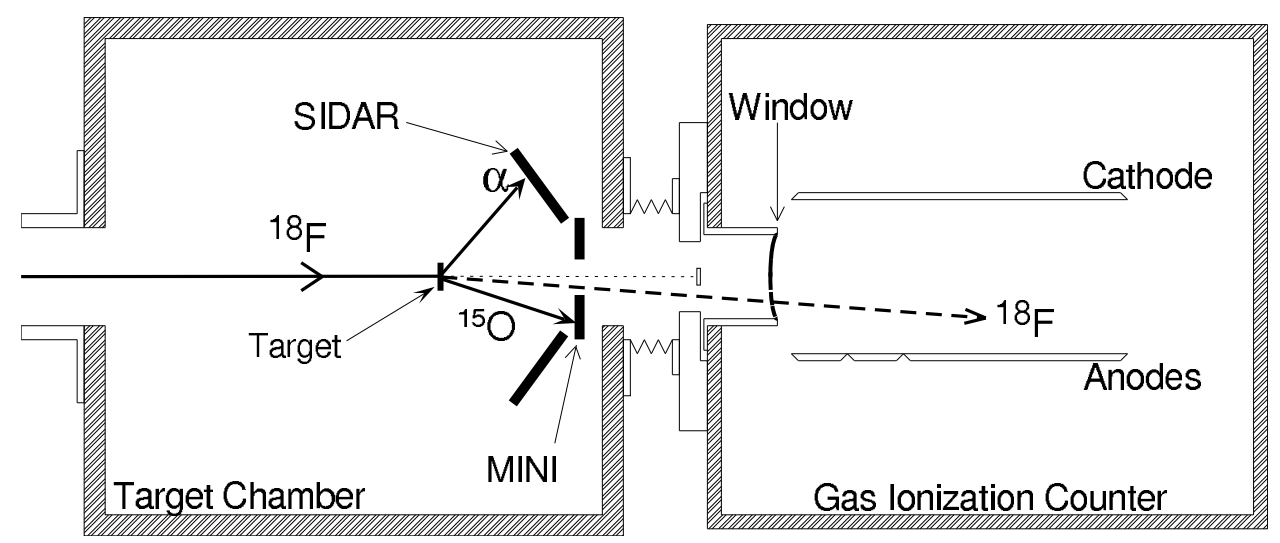

Figure 1: A schematic diagram of the experimental setup is shown.

\footnotetext{
* Speaker.
} 
[16]. The results show that four out of eight possibilities could be ruled out, and all four of the "allowed" possibilities produce nearly identical cross sections above the $665-\mathrm{keV}$ resonance. The allowed possibilities are $(+++),(+-+),(-++)$, and $(--+)$, where the signs in parenthesis are the signs of the 8-, 38-, and 665-keV resonances, respectively. We compare in Figure 2 the ${ }^{18} \mathrm{~F}(p$, $\alpha)^{15} \mathrm{O}$ excitation function to theoretical cross section calculations from the $R$-matrix code MULTI. Two cases of the relative signs are shown in the figure for illustration purposes. The theoretical cross sections were calculated over the complete range of energies and then averaged over the energy loss in the target as well as over the angles covered by the detectors $\left(56^{\circ} \leq \theta_{\text {c.m. }} \leq 138^{\circ}\right)$ for direct comparison with the data. Only upper limits on the cross section could be obtained at $E_{c . m .}=$ 770 and $824 \mathrm{keV}$ due to the large ${ }^{18} \mathrm{O}$ contamination of the beam $\left({ }^{18} \mathrm{~F} /{ }^{18} \mathrm{O} \sim 0.04\right)$. Since all four cases with a negative sign for the $665-\mathrm{keV}$ were ruled out, it is clear that the two resonances at $E_{c . m}$. $=8$ and $38 \mathrm{keV}$ do not strongly affect the cross section above $665-\mathrm{keV}$. Interference effects from these resonances, however, become more important at the lower energy range $\left(E_{c . m} \leq 600 \mathrm{keV}\right)$ as shown in Figure 3, where we show the astrophysical S-factor plots for 4 allowed possibilities.

New upper limits on the proton widths $\left(\Gamma_{p}\right)$ of the $E_{c . m .}=827$ and $842 \mathrm{keV}$ resonances have also been set. For a given set of resonance parameters [17], the upper limits on $\Gamma_{p}$ were calculated at $90 \%$ confidence level from the $\chi^{2}$ distribution. Upper limits were found to be $\Gamma_{p} \leq 1.17 \mathrm{keV}$ at $E_{c . m .}=827 \mathrm{keV}$ and $\Gamma_{p} \leq 1.65 \mathrm{keV}$ at $E_{c . m .}=842 \mathrm{keV}$, respectively. The upper limit at $E_{c . m .}=842$

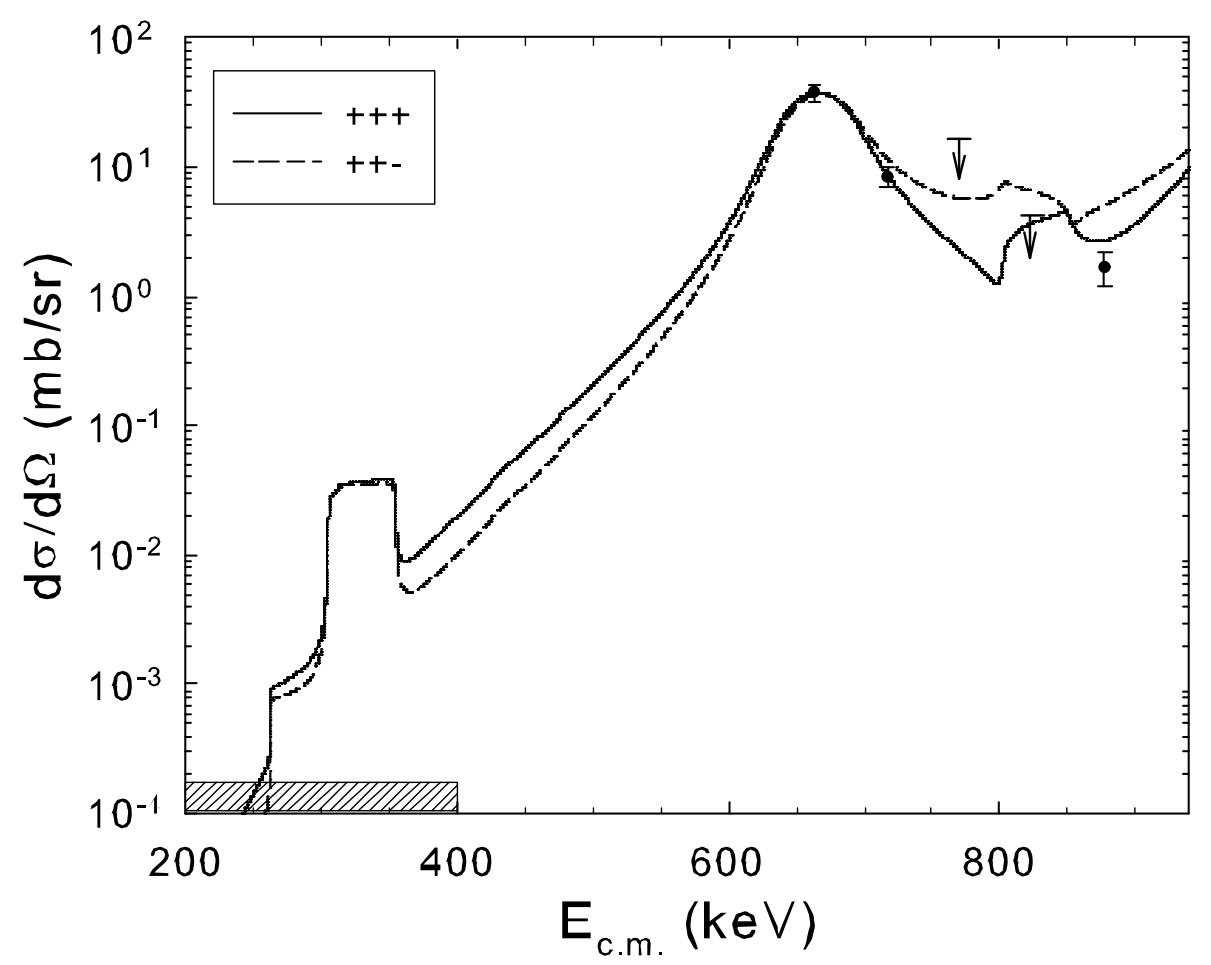

Figure 2: The ${ }^{18} \mathrm{~F}(p, \alpha){ }^{15} \mathrm{O}$ excitation function is shown along with theoretical cross section calculations from the $R$-matrix code MULTI. Most effective energy range for novae is indicated by the shaded box. 


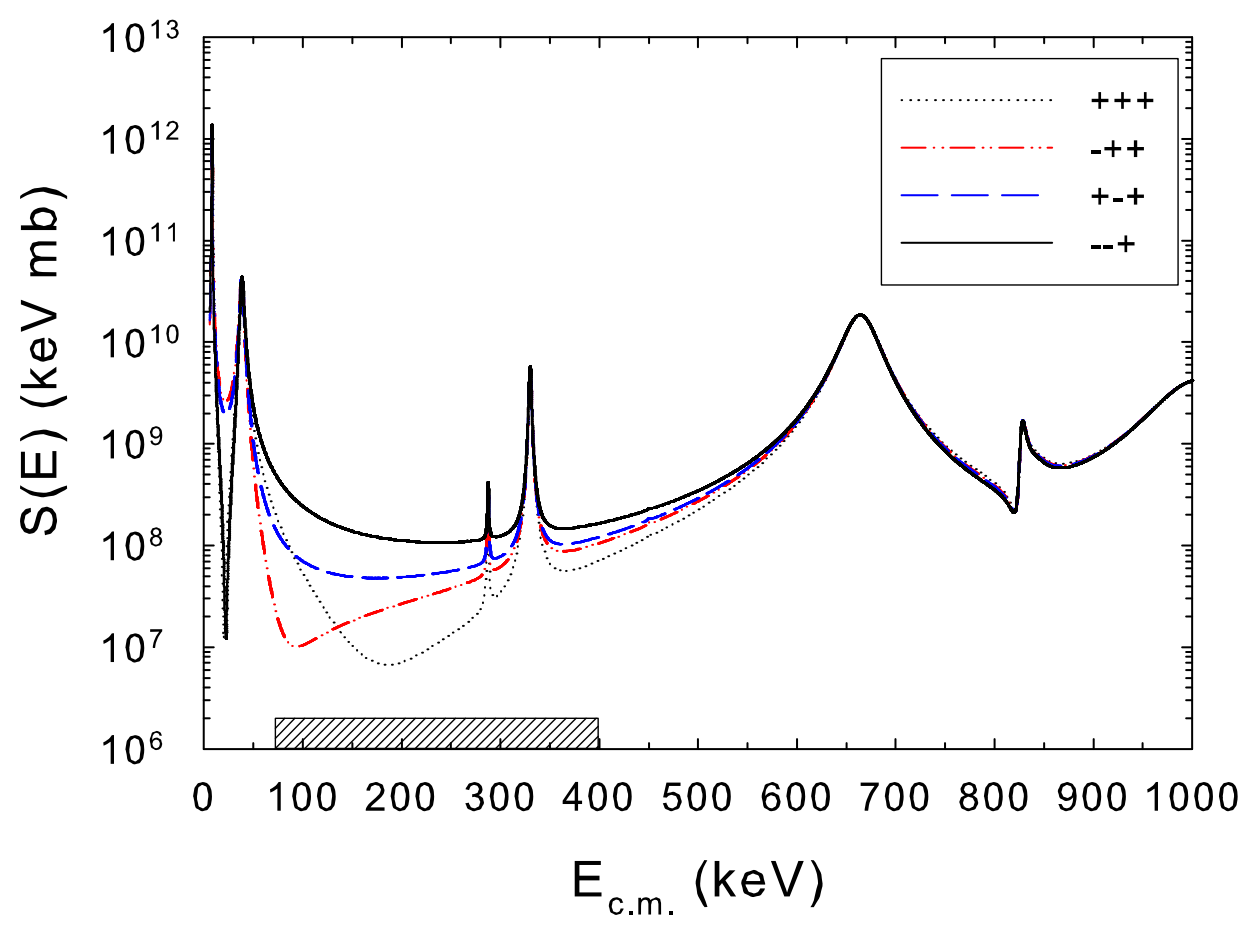

Figure 3: Astrophysical S-factor vs. center of mass energy plots for allowed four possibilities.

$\mathrm{keV}$ is consistent with the previously determined values from a ${ }^{18} \mathrm{~F}(p, p){ }^{18} \mathrm{~F}$ measurement in Ref. [10], while the other upper limit is less stringent than the previous one.

To investigate how this uncertainty in interference propagates to uncertainties in ${ }^{18} \mathrm{~F}$ production in novae, we have performed element synthesis calculations in the framework employed in the Computational Infrastructure for Nuclear Astrophysics [18], where the ejected envelope of nova is divided into 28 zones, each with its own thermodynamic history (time histories of the temperature and density). The result shows that the uncertainty in the ${ }^{18} \mathrm{~F}(p, \alpha){ }^{15} \mathrm{O}$ reaction rate due to the interference produces roughly a factor of 2 variation in the amount of ${ }^{18} \mathrm{~F}$ produced.

In conclusion, the ${ }^{18} \mathrm{~F}(p, \alpha){ }^{15} \mathrm{O}$ reaction rate was uncertain partly because of the lack of experimental knowledge about the relative signs of the interference of three $3 / 2^{+}$resonances. By measuring the ${ }^{1} \mathrm{H}\left({ }^{18} \mathrm{~F}, \alpha\right){ }^{15} \mathrm{O}$ cross sections in the energy range of $E_{c . m} .=663-877 \mathrm{keV}$ using radioactive ${ }^{18} \mathrm{~F}$ beams at the HRIBF, we provide the first experimental constraints on the interference effects. Our results show that the uncertainty in the reaction rate at the temperature range $0.3 \mathrm{GK}$ $\leq T \leq 0.6 \mathrm{GK}$ is reduced by up to $37 \%$ compared to previous work [11]. We also set new upper limits on proton widths at $E_{c . m .}=827 \mathrm{keV}\left(\Gamma_{p} \leq 1.17 \mathrm{keV}\right)$, and $E_{c . m .}=842 \mathrm{keV}\left(\Gamma_{p} \leq 1.65 \mathrm{keV}\right)$.

Oak Ridge National Laboratory is managed by UT-Battelle, LLC, for the U.S. Department of Energy under contract DE-AC05-00OR22725. This work was also supported in part by the U.S. Department of Energy under Contract Nos. DE-FG02-96ER40955 and DE-FG02-96ER40990 with Tennessee Technological University, and DE-FG03-93ER40789 with the Colorado School 
of Mines.

\section{References}

[1] A. Coc, M. Hernanz, J. José, and J.-P. Thibaud, Influence of new reaction rates on ${ }^{18} \mathrm{~F}$ production in novae, Astron. Astrophys. 357, 561 (2000).

[2] M. Hernanz, J. José, A. Coc, J. Gómez-Gomar, and J. Isern, GAMMA-RAY EMISSION FROM NOVAE RELATED TO POSITRON ANNIHILATION: CONSTRAINTS ON ITS OBSERVABILITY POSED BY NEW EXPERIMENTAL NUCLEAR DATA, Astrophys. J. 526, L97 (1999).

[3] S. Utku et al., Breakout from the hot CNO cycle: The ${ }^{18} F(p, \gamma) v s{ }^{18} F(p, \alpha)$ branching ratio, Phys. Rev. C 57, 2731 (1998).

[4] R. Coszach et al., A direct measurement of the ${ }^{18} F(p, \alpha)^{15} O$ reaction, Phys. Lett. B 353, 184 (1995).

[5] K. E. Rehm et al., Astrophysical reaction rate for the ${ }^{18} F(p, \alpha)^{15}$ O reaction, Phys. Rev. C 53, 1950 (1996).

[6] D. W. Bardayan et al., Kinematically complete measurement of the ${ }^{1} H\left({ }^{18} \mathrm{~F}, p\right){ }^{18} \mathrm{~F}$ excitation function for the astrophysically important 7.08-MeV state in ${ }^{19} \mathrm{Ne}$, Phys. Rev. C 62, 042802(R) (2000).

[7] D. W. Bardayan et al., Destruction of ${ }^{18} \mathrm{~F}$ via ${ }^{18} \mathrm{~F}(\mathrm{p}, \alpha){ }^{15} \mathrm{O}$ burning through the $E_{c . \mathrm{m} .}=665 \mathrm{keV}$ resonance, Phys. Rev. C 63, 065802 (2001).

[8] N. de Séréville, E. Berthoumieux, and A. Coc, The ${ }^{18} F(p, \alpha){ }^{15} O$ reaction rate for application to nova $\gamma$-ray emission, Nucl. Phys. A 758, 745c (2005).

[9] D. W. Bardayan et al., Strength of the ${ }^{18} F(p, \alpha)^{15} O$ Resonance at $E_{c . m .}=330 \mathrm{keV}$, Phys. Rev. Lett. 89, 262501 (2002).

[10] D. W. Bardayan, J. C. Blackmon, J. Gómez del Campo, R. L. Kozub, J. F. Liang, Z. Ma, L. Sahin, D. Shapira, and M. S. Smith, Search for astrophysically important ${ }^{19}$ Ne levels with a thick-target ${ }^{18} F(p, p){ }^{18}$ F measurement, Phys. Rev. C 70, 015804 (2004).

[11] R. L. Kozub et al., New constraints on the ${ }^{18} F(p, \alpha){ }^{15} O$ rate in novae from the $(d, p)$ reaction, Phys. Rev. C 71, 032801(R) (2005).

[12] D. W. Stracener, Status of radioactive ion beams at the HRIBF, Nucl. Instrum. Methods Phys. Res., Sect. B 204, 42 (2003).

[13] G. D. Alton and J. R. Beene, The Holifield Radioactive Ion Beam Facility at the Oak Ridge National Laboratory: Present status and future plans, J. Phys. G: Nucl. Part. Phys. 24, 1347 (1998).

[14] R. F. Welton et al., The development of the ${ }^{17} \mathrm{~F}$ beam at the Holifield radioactive ion beam facility, Nucl. Phys. A 701, 452c (2002).

[15] R. O. Nelson, E. G. Bilpuch, and G. E. Mitchell, ANALYSIS OF PROTON RESONANCE SCATTERING FROM NON-ZERO SPIN TARGETS, Nucl. Instrum. Methods Phys. Res., Sect. A 236, $128(1985)$.

[16] A. M. Lane and R. G. Thomas, R-matrix Theory of Nuclear Reactions, Rev. Mod. Phys. 30, 257 (1958).

[17] K. Y. Chae et al., First experimental constraints on the interference of $\frac{3}{2}^{+}$resonances in the ${ }^{18} F(p, \alpha){ }^{15} O$ reaction, Phys. Rev. C 74, 012801(R) (2006).

[18] Computational Infrastructure for Nuclear Astrophysics, http://www.nucastrodata.org. 